\title{
Acquisition of leverpressing without assistance by rats maintained on interval and ratio schedules
}

\author{
LAURENCE MILLER and DANIEL C. LINWICK \\ Western Washington University, Bellingham, Washington 98225
}

\begin{abstract}
Rats can acquire a leverpressing response reinforced with food according to continuous or differential reinforcement of low rate schedules without experimenter assistance. In the present study, acquisition of responding without assistance on fixed- and variable-interval and fixedand variable-ratio schedules was examined. The intervals employed were 30 and $180 \mathrm{sec}$, and the ratio requirement was 10 responses per reinforcer. Fourteen of 15 rats on the interval schedules acquired and maintained leverpressing, while only 2 of 8 rats on the ratio schedules did so.
\end{abstract}

Studies have shown rats to be capable of acquiring a leverpressing response for food without magazine training or experimenter assistance of any kind. This acquisition has been demonstrated with food presented according to simple (continuous reinforcement) or complex (differential reinforcement of low rates, DRL) schedules (Kopp, Bourland, Tarte, \& Vernon, 1976; Linwick \& Miller, 1978; Miller, 1976).

In the present study, acquisition of responding maintained by fixed-and variable-interval (FI and $\mathrm{VI}$ ) and by fixed- and variable-ratio (FR and VR) schedules without prior magazine training or assistance was examined. Two interval durations were employed, 30 and $180 \mathrm{sec}$. Since rats are capable of acquiring leverpressing with a DRL 30-sec schedule in effect, one might expect that responding on FI and VI $30 \mathrm{sec}$ might also be readily acquired. Of particular interest was whether responding would be acquired with the long interval duration of $180 \mathrm{sec}$.

The present experiment also attempted to determine whether rats are capable of acquiring leverpressing on ratio schedules without experimenter assistance. Schedules with a clear temporal component, such as DRL, FI, and VI, may be contrasted with ratio schedules that lack such a component. Thus, to receive reinforcement, a rat on a DRL or interval schedule need only emit a single response, given an appropriate period of time has elapsed since the previous response, whereas a rat on a ratio schedule must emit a specified number of responses. Hence, a "small" ratio requirement of 10 responses per reinforcer was chosen.

We thank Will Neill, Mike Hagarty, and Don Martin for their assistance in running rats. Reprints may be obtained from Laurence Miller, Department of Psychology, Western Washington University, Bellingham, Washington 98225.

\section{METHOD}

\section{Subjects}

Twenty-three experimentally naive male rats, about 180 days old, were deprived to $80 \%$ of their free-feeding weights over a 7-day period prior to the start of the experiment.

\section{Apparatus}

Standard single-lever Grason-Stadler operant chambers were enclosed in sound-attenuating chests.

\section{Procedure}

When subjects reached $80 \%$ of their free-feeding weights, they were placed in the operant chamber for the first time ever. Subjects on interval schedules were presented with FI 30-sec, FI 180-sec, VI 30-sec (3- to 57 -sec interval range), or VI $180 \mathrm{sec}$ (10- to 350-sec range) schedules ( $n=4$ subjects, exœpt for VI $180 \mathrm{sec}, \mathrm{n}=3$ ). The remaining subjects were presented with either a FR 10 or a VR 10 schedule $(n=4$ subjects for each schedule). The response requirement varied from 1 to 22 responses for the VR schedule. Notably, the first response emitted on the VR 10 schedule at the beginning of a session always produced a reinforcer. For both interval and ratio schedules, a single $45-\mathrm{mg}$ Noyes pellet was used as the reinforcer. All subjects received 15 daily sessions, each $40 \mathrm{~min}$ long. The number of responses emitted and reinforcers received during each session was recorded. The number of sessions required to reach a criterion of 10 reinforced responses in a single session was also recorded. All subjects failing to reach criterion in the 15 sessions allotted were assigned a maximum value of 15 .

\section{RESULTS}

The number of responses emitted and reinforcers received by the subjects in the interval and ratio conditions are presented in Figures 1-3, respectively. Only data for those subjects with the ratio schedules that acquired leverpressing are presented. Notably, all of the animals except one in the interval conditions acquired and maintained leverpressing, while only two of the ratio animals did. Also, with the interval schedules, although individual rates of responding 


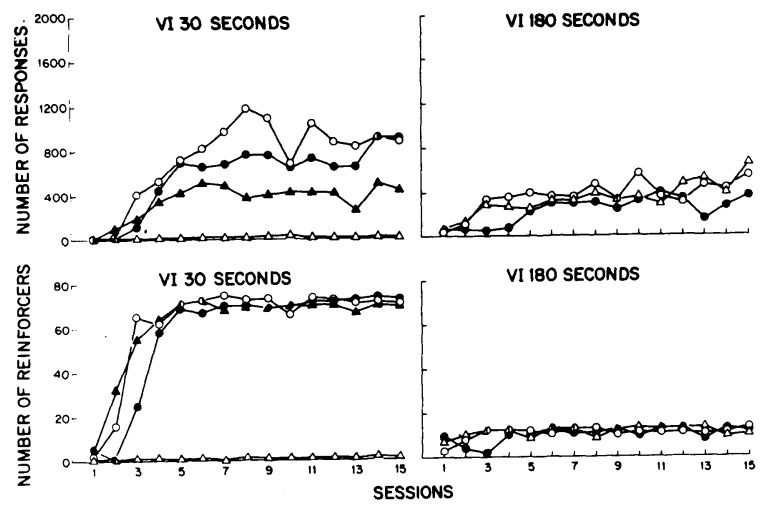

Figure 1. Number of responses emitted and number of reinforcers received by each animal on each VI schedule for the 15 test sessions.

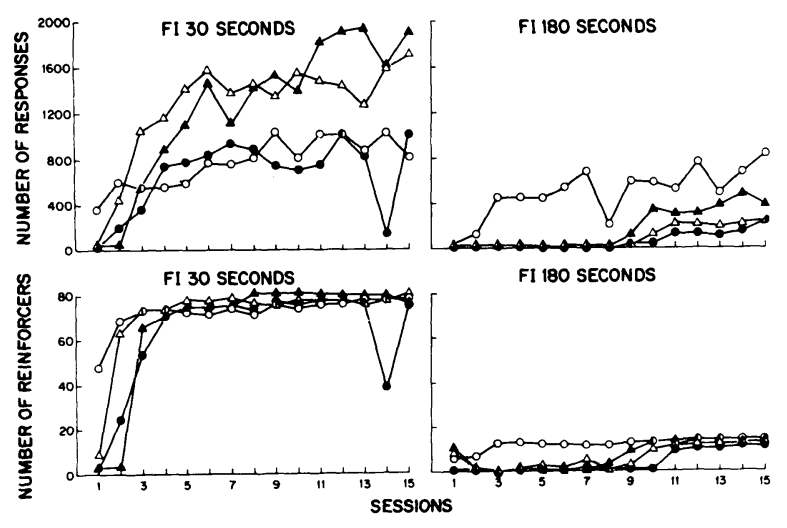

Figure 2. Number of responses emitted and number of reinforcers received by each animal on each FI schedule for the 15 test sessions.

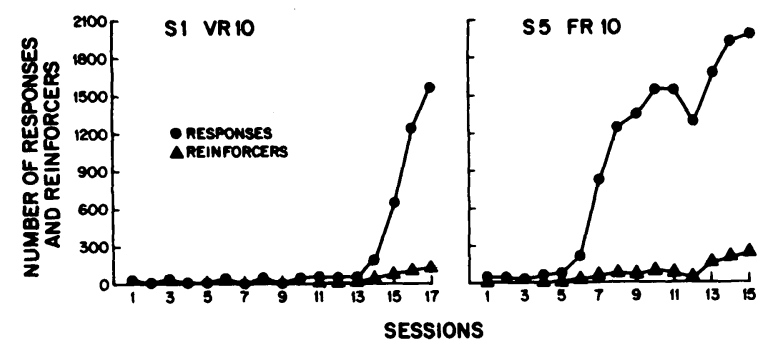

Figure 3. Number of responses emitted and number of reinforcers received by each successful animal on FR 10 and VR 10 for the 15 test sessions.
Table 1

Mean Sessions to Criterion (10 Reinforced Responses in a Single Session) for Groups FI and VI 30 and $180 \mathrm{sec}$ and for Groups FR 10 and VR 10

\begin{tabular}{|c|c|c|c|c|c|}
\hline \multicolumn{2}{|c|}{ FI } & \multicolumn{2}{|c|}{ VI } & \multirow[b]{2}{*}{ FR 10} & \multirow[b]{2}{*}{ VR 10} \\
\hline 30 & 180 & 30 & 180 & & \\
\hline 2.0 & 6.5 & 5.5 & 2.0 & 12.8 & 14.8 \\
\hline
\end{tabular}

varied, all animals that acquired responding obtained about the same number of reinforcers per session, which was very near the maximum possible.

Table 1 presents the sessions-to-criterion data for the various groups. An analysis of variance of these data revealed a significant sessions effect $[F(5,17)=8.20$, $\mathrm{p}<.05]$. A Newman-Keuls post hoc analysis indicated that none of the interval groups differed from the others. Moreover, the two ratio groups also failed to differ from each other. Each interval group required fewer sessions to reach the 10 reinforced responses criterion than each of the ratio groups $(\mathrm{p}<.05)$.

\section{DISCUSSION}

The performance of animals on interval schedules of reinforcement stood in direct contrast to that of animals on ratio schedules. These findings suggest the relevance of a temporal dimension to ease of response acquisition in the absence of experimenter assistance. Whereas rats on interval schedules need only to emit a single response to receive the reinforcer, this is clearly not the case for rats on ratio schedules. It therefore seems likely that the ease of acquisition of leverpressing by a naive animal on a ratio schedule is directly related to its preconditioning operant baseline level of responding.

\section{REFERENCES}

Kopp, J., Bourland, G., Tarte, R. D., \& Vernon, C. R. Acquisition of bar pressing in nondeprived rats. Psychological Record, 1976, 26, 49-54.

LINwICK, D. C., \& MilleR, L. Acquisition of leverpressing without experimenter assistance by rats on differential reinforcement of low rates schedules. Bulletin of the Psychonomic Society, 1978, 12, 193-195.

MrLlER, L. Acquisition of lever pressing by rats with and without magazine training. Psychological Record, 1976, 26, 355-359.

(Received for publication November 29, 1978.) 